\title{
Progressive Metabolic and Structural Cerebral Perturbations After Traumatic Brain Injury: An In Vivo Imaging Study in the Rat
}

\author{
Ying R. Liu* ${ }^{* 1}$, Lisa Cardamone*1, R. Edward Hogan ${ }^{2}$, Marie-Claude Gregoire ${ }^{3}$, John P. Williams ${ }^{4}$, Rod J. Hicks ${ }^{5}$, \\ David Binns $^{5}$, Amelia Koe ${ }^{1}$, Nigel C. Jones ${ }^{1}$, Damian E. Myers ${ }^{1}$, Terence J. O’Brien ${ }^{1}$, and Viviane Bouilleret ${ }^{1,6}$ \\ ${ }^{1}$ Department of Medicine, The Royal Melbourne Hospital, University of Melbourne, Victoria, Australia; ${ }^{2}$ Department of Neurology, \\ Washington University, St. Louis, Missouri; ${ }^{3}$ Australian Nuclear Science and Technology Organisation, New South Wales, Australia; \\ ${ }^{4}$ Small Animal MRI Facility, Florey Neurosciences Institute, Victoria, Australia; ${ }^{5}$ Centre for Molecular Imaging, Peter MacCallum \\ Cancer Centre, Victoria, Australia; and ${ }^{6}$ Department of Neurophysiology and Epilepsy, APHP, CHU Bicêtre, Paris, France
}

Traumatic brain injury (TBI) has a high incidence of long-term neurologic and neuropsychiatric morbidity. Metabolic and structural changes in rat brains were assessed after TBI using serial ${ }^{18} \mathrm{~F}-\mathrm{FDG}$ PET and 3-dimensional MRI in vivo. Methods: Rats underwent lateral fluid percussion injury (FPI; $n=16$ ) or a sham procedure $(n=11)$. PET and MR images were acquired at $1 \mathrm{wk}$ and at 1,3 , and 6 mo after injury. Morphologic changes were assessed using MRI-based regions of interest, and hippocampal shape changes were assessed with large-deformation highdimensional mapping. Metabolic changes were assessed using region-of-interest analysis and statistical parametric mapping with the flexible factorial analysis. Anxiety-like behavior and learning were assessed at 1, 3, and 6 mo after injury. Results: PET analyses showed widespread hypometabolism in injured rats, in particular involving the ipsilateral cortex, hippocampus, and amygdalae, present at $1 \mathrm{wk}$ after FPI, most prominent at $1 \mathrm{mo}$, and then decreasing. Compared with the sham group, rats in the FPI group had decreased structural volume which progressively increased over 3-6 mo, occurring in the ipsilateral cortex, hippocampus, and ventricles after FPI $(P<0.05)$. Largedeformation high-dimensional mapping showed evolving hippocampal shape changes across the 6 mo after FPI. Injured rats displayed increased anxiety-like behavior $(P<0.05)$, but there were no direct correlations between the severity of the behavior abnormalities and functional or structural imaging changes. Conclusion: In selected brain structures, FPI induces early hypometabolism and delayed progressive atrophic changes that are dynamic and continue to evolve for months. These findings have implications for the understanding of the pathophysiology and evolution of long-term neurologic morbidity following TBI, and indicate an extended window for targeted neuroprotective interventions.

Key Words: traumatic brain injury; rat; ${ }^{18}$ F-FDG PET; MRI; anxiety

J Nucl Med 2010; 51:1788-1795

DOI: 10.2967/jnumed.110.078626

Received Apr. 30, 2010; revision accepted Aug. 9, 2010.

For correspondence or reprints contact: Viviane Bouilleret, $\mathrm{CHU}$ Bicêtre, UNCE, Department of Neurophysiology and Epilepsy, 78, Rue du Général Leclerc, 94275 Le Kremlin-Bicetre Cedex, Paris, France.

E-mail: viviane.bouilleret@bct.aphp.fr

${ }^{*}$ Contributed equally to this work.

COPYRIGHT @ 2010 by the Society of Nuclear Medicine, Inc.
$\mathbf{P}$ ET with ${ }^{18} \mathrm{~F}-\mathrm{FDG}$ is a powerful imaging technique that can map regional cerebral metabolism patterns in vivo in humans and animals. Cross-sectional studies using ${ }^{18} \mathrm{~F}-\mathrm{FDG}$ PET in humans at various times after severe traumatic brain injury (TBI) suggest a triphasic pattern of perturbations in metabolic brain function (1-3). Acutely after TBI, an elevated metabolic state occurs. This initial brief response is followed by a relatively prolonged period of metabolic suppression before ${ }^{18} \mathrm{~F}$-FDG uptake normalizes to baseline levels. However, there are discrepancies between the reports, and ${ }^{18} \mathrm{~F}$ FDG PET use for TBI remains controversial. Some studies report a return to baseline ${ }^{18} \mathrm{~F}-\mathrm{FDG}$ uptake within $1 \mathrm{mo}$, regardless of the severity of the trauma (4), whereas others found metabolic abnormalities that persist chronically. Finally, across different patients involving chronic stages of trauma (at least 3 mo after TBI) both regional hypometabolism and regional hypermetabolism have been reported for the same regions (5). Because of radiation dosimetry issues, no long-term follow-up studies with serial ${ }^{18}$ F-FDG PET have been performed in humans after TBI. Therefore, the longterm evolution and outcome of these acute metabolic changes in individuals is uncertain, as is their relationship to the longterm structural brain changes that are also commonly present.

Here, we utilized serial in vivo imaging with small-animal PET and small-animal MRI to identify and track the spatial and temporal evolution of functional and structural changes in the brain for 6 mo after lateral fluid percussion injury (FPI) in the rat. One previous study used ${ }^{18} \mathrm{~F}$-FDG PET to assess metabolic changes after TBI in rats but had only a 10-d follow-up and provided no assessment of structural changes (6). Several studies have used small-animal MRI to assess long-term morphologic changes after TBI in rats, demonstrating atrophy in the cortex and hippocampus similar to findings observed in humans (7-9). However, none of these studies incorporated concurrent metabolic or functional changes. Lateral FPI induces both focal and diffuse cerebral injury and is a widely accepted experimental model of moderate-to-severe human closed-head TBI $(10,11)$. The severity of the imag- 
ing changes was correlated with quantitative measures of anxiety-like behavior, because mood disorders (including hyperanxiety) are common in patients after TBI, and we have demonstrated long-term increases in anxiety-like behavior in rats after FPI $(7,12)$.

\section{MATERIALS AND METHODS}

\section{Animal Housing}

Male Wistar rats $(n=27)$, aged between 10 and $17 \mathrm{wk}$ and weighing $281.2 \pm 7.8 \mathrm{~g}$ at the beginning of the experiment, were used. Rats were bred onsite at the Royal Melbourne Hospital and individually housed under controlled temperatures of $22^{\circ} \mathrm{C} \pm 1{ }^{\circ} \mathrm{C}$, in a 12-h light-dark cycle, on at 6 AM, with water and food chow ad libitum. All experimental procedures were approved by the University of Melbourne Animal Ethics Committee (AEC 0705687).

\section{Lateral FPI Model}

TBI was induced using lateral FPI based on standard descriptions (10) with minor modifications, as previously described $(7,12)$. Briefly, under anesthesia a 5-mm craniotomy, centered $4 \mathrm{~mm}$ lateral and $4 \mathrm{~mm}$ posterior to the bregma, was performed. The left edge of the craniotomy was adjacent to the left lateral ridge, to induce a lateral injury (13). A dissecting microscope was used to examine the dura to ensure there was no bleeding from drilling. A severeintensity pressure pulse (amplitude of 3.2-3.5 atm (13)) was delivered by a fluid-percussion device via a modified female Luer-Lock (Terumo) cap. The lateral position of cortical injury minimized direct involvement of the hippocampus, with this and other structures being secondarily affected by the transmitted force, modeling closed-head injury in patients. The sham group underwent the same surgical procedures, without application of the fluid pulse.

Acute neurologic injury was assessed in all rats on the day before and for $3 \mathrm{~d}$ after FPI using a composite neuromotor score adapted from McIntosh et al. (13), as previously described $(7,12)$. Each of the 10 tasks was rated as 0 (pass) or 1 (fail), for a maximum total score of 10 .

\section{PET Acquisition}

Serial ${ }^{18}$ F-FDG PET scans were obtained (FPI, $n=16$; sham, $n=$ 11) at $1 \mathrm{wk}$ and at 1,3 , and 6 mo after FPI or sham injury. PET images were acquired using a Mosaic Animal PET scanner (Philips) at the small-animal PET facility at the Centre for Molecular Imaging, Peter MacCallum Cancer Institute. PET scans were acquired 1-3 d before the corresponding MRI scans. Rats were injected intraperitoneally with $37-74 \mathrm{MBq}(1-2 \mathrm{mCi})$ of ${ }^{18} \mathrm{~F}-\mathrm{FDG}$ at $30 \mathrm{~min}$ before scanning. Subsequently, rats were anesthetized with $2 \%$ isoflurane (1:1 oxygen:air) and placed prone onto an acrylic platform for a 30-min acquisition.

Corrections were applied for dead-time loss, decay, and activity injected (standardized uptake value $[\mathrm{kBq} / \mathrm{mL}])$. No corrections were made for attenuation or scatter. The reconstruction algorithm was an ordered-subset expectation maximization iterative protocol (4 iterations and 8 subsets). The intrinsic reconstructed resolution of the system was $2.1 \mathrm{~mm}$ in full width at half maximum at the center of the field of view, with one hundred twenty $128 \times 128$ transaxial slices and a $1 \times 1 \times 1 \mathrm{~mm}$ voxel size.

\section{PET Region-of-Interest (ROI) Analysis}

Each ${ }^{18} \mathrm{~F}$-FDG PET image was manually coregistered to its corresponding MRI scan (i.e., same rat at the same time point), on which 11 ROIs were defined (Supplemental Fig. 1; supplemental materials are available online only at http://jnm.snmjournals. org). Coregistration was based on manual identification of 6 landmarks (Harderian glands, olfactory bulbs, cerebral cortex, and cerebellum) by a single operator unaware of rat number, injury status, or imaging time point. Nine random points were selected within the original locations in the PET volume. We have previously demonstrated this coregistration method to be more accurate than the automated algorithms, mutual information, and surface matching, likely because of the lack of anatomic information in the PET images (14). To validate the accuracy of our coregistration, a test set of PET/MR images, for which the rats had fiducial markers that were visible on MR and PET images subcutaneously implanted, were manually coregistered by 2 masked users 3 times (Supplemental Fig. 1C). The validation had 2 components: registration error of coordinates of 9 points compared with perfect registration, determined using the subcutaneous fiducial markers (mean difference, $0.15 \mathrm{~mm}$; 95\% confidence interval, 0.56-2.57 $\mathrm{mm})$ and interoperator error, the mean difference of coordinates for the 9 points between 2 users $(1.2 \mathrm{~mm}$; $95 \%$ confidence interval, $0.144-2.3 \mathrm{~mm}$ ). The mean activity was measured for all $11 \mathrm{ROIs}$, and mean value of activity for the ROIs was calculated. The cerebellum was outlined on the MR image and its mean calculated activity used as the reference for normalization of PET data. There was no statistically significant difference in cerebellar standardized uptake value at any time point between the FPI and sham rats, nor over time within either of the groups, indicating that it is an appropriate, metabolically stable reference region for the PET image normalization in this study. The normalization of intensity for each ROI was calculated as:

$$
\frac{\text { ROI activity }(\text { per } \mathrm{mL})}{\text { Cerebellum activity }(\text { per } \mathrm{mL})} \times 100 .
$$

\section{PET Statistical Parametric Mapping (SPM) Analysis}

We used SPM (SPM5; Welcome Department of Cognitive Neuroscience) to process the PET data as follows.

For PET preprocessing, the PET subvolumes encompassing the rat brain, excluding extracerebral activity, were extracted and resampled to a $0.11718 \mathrm{~mm}^{3}$ voxel size into the 3-dimensional space of a reference MR image using trilinear interpolation (Analyze; Mayo Foundation).

As a reference PET image, we chose 1 control (sham) PET scan with a high degree of symmetry, alignment, and freedom from obvious artifacts. Following the work of Casteels et al. (15), we realigned 6 other individual PET scans from rats of similar weight (1 baseline PET and 5 from the first-week sham group) using a rigid transformation (6 parameters) and trilinear interpolation to the reference PET image to create a mean image for use as the PET template.

To account for brain size variations over time, all individual PET images were spatially normalized to the PET template using the affine spatial normalization option in SPM and a trilinear interpolation. The voxel size of the normalized and resampled PET images was $0.1 \mathrm{~mm}^{3}$.

Flexible factorial analysis with 3 independent factors was applied: treatment (sham or FPI), time (1 wk and 1, 3, or 6 mo), and subject (rat number), with an interaction between factors 1 and 2 to perform the analysis. The PET images were proportionally normalized using the cerebellum mean value derived from the manual analysis. Contrasts assessing differences between sham and FPI at each time point were derived and tested at a $P$ value of less than 0.01 (falsediscovery rate [FDR] error metric). The resolution element size was $4.6 \times 4.1 \times 5.0 \mathrm{~mm} ; \mathrm{T}$ maps contained 2,010,067 voxels. 


\section{MR Image Acquisition}

T2-weighted small-animal MRI scans were obtained at baseline, $1 \mathrm{wk}$, and 1, 3, and 6 mo after FPI. Small-animal MRI data were acquired using a 4.7-T 47/30 Advance small-animal spectrometer in a Paravision 3.0 console (Bruker Biospec), with a shield-gradient set appropriately adjusted for rats (16).

\section{Anatomic Structure Delineation (ROI Analysis)}

MRI volumes of selected brain regions were quantified with manually drawn ROIs using Analyze. ROIs were drawn on consecutive axial MRI slices by an investigator unaware of the rats' injury status (Supplemental Fig. 1A). Only slices containing hippocampus (average of 11 slices; Supplemental Fig. 1B) were analyzed. Before ROIs were drawn, the MR image set was coregistered to a template MR image with the ROIs predefined. The ROIs were then redrawn for the target MR image on the basis of this template. Eleven ROIs were drawn identically as previously described (16): the cortex, hippocampus, thalamus, hypothalamus, amygdala, and lateral ventricles from both hemispheres.

\section{Large-Deformation High-Dimensional Mapping (HDM-LD) of Hippocampal Morphometry}

HDM-LD utilizes the power of computer-assisted shape recognition to identify patterns within image data $(16,17)$. Computational anatomic techniques produce 3-dimensional surface representations of the hippocampus with resolution at a subvoxel level, enabling visualization of details of hippocampal surface anatomy (18). Changes in morphology can be detected even when total hippocampal volume changes are not significantly different (19-22), using either visual analysis of deformation surfaces or multivariate ANOVA on the coefficients from eigenvector fields (23). Originally developed for human studies, this method has been adapted and validated for the assessment of rat hippocampus $(16,17)$. Methods for comparing groups were as previously described (16). In brief, for this study we generated coordinates for hippocampal surface transformation for each image, which in turn was used to generate an average hippocampal deformation surface at baseline, $1 \mathrm{wk}$, and 1 , 3 , and 6 mo scans in both the FPI and the sham groups. For final results, we calculated a minimum mean squared error estimation by coregistering the FPI and sham average hippocampal surfaces for each time point. By calculating differences in hippocampal transformation coregistration using the minimum mean squared error, we directly compared the shape of the hippocampi between the FPI and sham groups. Final results were projected on the sham group hippocampal surfaces. To define shape changes in the hippocampus, we assigned a color map, which represents the distance between the matched hippocampi. The maximum and minimum values of the color map were set using the maximum value of inward deformation. To complement the statistically confirmed findings of progressive atrophy from volumetric measurements of the hippocampi, the deformation patterns were visually compared between FPI and sham rats. Subfield involvement was determined by comparing regions of maximal deformation with a standard rat hippocampal subfield atlas (24).

\section{Behavioral Assessments}

At 1, 3, and 6 mo after injury on separate days, beginning at $10 \mathrm{Am}$, all rats underwent 2 well-validated assessments of anxiety-like behavior (open-field test and elevated plus maze [EPM]) and a test of learning and memory (Morris water maze). For each test, rats were brought into the behavioral testing facility at the Royal Melbourne Hospital at least $30 \mathrm{~min}$ before beginning the test.
Open-Field Test and EPM. The open-field test and EPM are widely used to assess anxiety-like behavior in rodents $(25,26)$, and procedures have been published previously by our group (12).

Morris Water Maze. The Morris water maze is a test widely used to assess spatial learning and memory in rodents; the procedure has been previously published by our group (27).

\section{Data Analyses and Statistics}

The neuromotor scoring data were not normally distributed (Kolmogorov-Smirnov goodness-of-fit test) and were therefore analyzed with the Friedman ANOVA for repeated measures (days after FPI), with injury status as the independent variable. The MRI and PET data for each ROI were analyzed separately using a 2-way ANOVA for repeated measures, with injury status and time after FPI as the independent variables. For regions in which an overall significant difference was found between the FPI and sham rats, Bonferroni post hoc planned-comparison analysis was used to cross-sectionally compare individual time points. Behavioral tests were analyzed using 2-way ANOVA, with injury status and time after FPI as the independent variables. Pearson correlations were used to assess the relationship between the magnitude of changes in the imaging variable and behavioral variables (where there was a difference between FPI and sham rats). All analyses were performed using Statistica software (StatSoft Inc.). Statistical significance was set at a $P$ value of 0.05 or less, and all data were expressed as mean \pm SEM unless otherwise stated.

\section{RESULTS}

\section{FPI Induces Subacute Neuromotor Deficits}

Average intensity of the fluid pulse delivered to the rats in the FPI group was $3.40 \pm 0.03$ atm. Compared with sham rats, major subacute neuromotor scoring deficits were observed in FPI rats $\left(\chi_{F(3,27)}^{2}=26.20, P<0.001\right.$; Supplemental Fig. 2), persisting for $3 \mathrm{~d}$ after FPI.

\section{ROI Analysis of ${ }^{18}$ F-FDG PET Images Demonstrates Regional Decreases in Brain Metabolism After FPI, Which Evolve Over Time}

Overall differences between sham and FPI rats were found for 3 ROIs on the PET scans: left cortex $\left(F_{2,24}=5.42, P=\right.$ $0.03)$, left hippocampus $\left(F_{2,24}=7.27, P=0.01\right)$, and left amygdala $\left(F_{2,24}=10.5, P=0.004\right)$ (Fig. 1$)$. An overall interaction with time was found for the left cortex $\left(F_{2,24}=\right.$ 4.64, $P=0.006)$. Specifically, the ipsilateral cortex exhibited significantly lower ${ }^{18} \mathrm{~F}$-FDG uptake $(16.6 \%)$ in FPI rats 3 mo after injury $(P=0.01$; Fig. $1 \mathrm{~A}) .{ }^{18} \mathrm{~F}-\mathrm{FDG}$ uptake was reduced in the ipsilateral hippocampus at all time points, with a mean difference of $-14.7 \%$. This difference reached statistical significance at $1 \mathrm{wk}$ and 3 mo (both $P=0.02$; Fig. 1B). In the ipsilateral amygdala, there was a significant reduction in ${ }^{18} \mathrm{~F}-\mathrm{FDG}$ uptake in FPI rats at $1(P=0.007)$ and $6(P=0.03)$ mo after injury, compared with the sham group (Fig. 1C).

\section{SPM Analysis of Serial ${ }^{18}$ F-FDG PET Images Demonstrates Topographic Progression of Hypometabolic Changes Over Time After FPI}

SPM analysis was performed on 6 sham and 6 FPI rats that had good-quality ${ }^{18}$ F-FDG PET scans for every time 


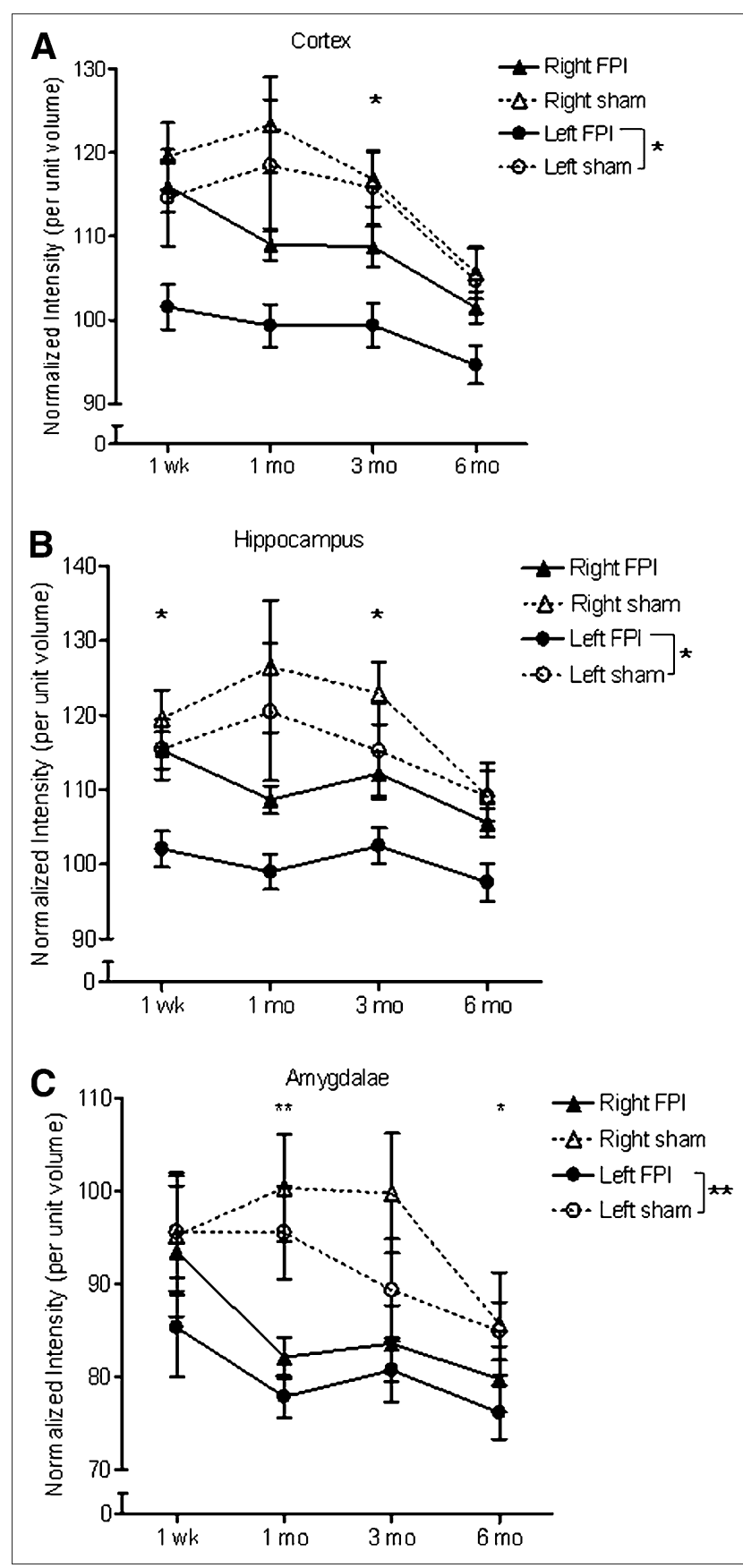

FIGURE 1. Time course of metabolic changes on PET scans in cortex (A), hippocampus (B), and amygdala (C) ROls. ${ }^{*} P<0.05$. ${ }^{* *} P<0.005$. (2-way ANOVA for repeated measures with Bonferroni post hoc analysis; mean \pm SEM.)

point (Fig. 2). One week after injury, a significant and extended region of cortical hypometabolism was demonstrated (cluster level: voxels per cluster $\left[\mathrm{k}_{\mathrm{E}}\right]=672,802$ voxels, $P$ corrected $<0.001)$. Two subclusters could be outlined: the anterior part of the left cortex, extending to involve the left striatum (probability of the FDR $\left[\mathrm{P}_{\mathrm{FDR}}\right]$ corrected $=0.002 ; z=4.5$ ), and the posterior part of the right cortex, above the superior part of the hippocampi
$\left(\mathrm{P}_{\mathrm{FDR}}\right.$ corrected $\left.=0.002 ; z=4.28\right)$. One month after FPI, the volume of the marked anterior left cortical hypometabolism had dramatically reduced, no longer including the striatum $\left(\mathrm{k}_{\mathrm{E}}=84,138 ; P\right.$ corrected $=0.007 ; \mathrm{P}_{\mathrm{FDR}}$ corrected $=0.009 ; z=4.54$; Fig. 2A). Moreover, this region of hypometabolism shifted toward more posterior structures to involve the left hippocampus, left amygdala, and left posterior cortex $\left(\mathrm{k}_{\mathrm{E}}=138,760 ; P\right.$ corrected $=$ 0.001; $\mathrm{P}_{\mathrm{FDR}}$ corrected $=0.009 ; z=4.49$; Fig. $2 \mathrm{C}$ ). On the contralateral side, the significant hypometabolic regions shifted toward the posterior lateral cortex, extending to involve the amygdala $\left(\mathrm{k}_{\mathrm{E}}=123,515 ; P\right.$ corrected $=$ 0.002; $\mathrm{P}_{\mathrm{FDR}}$ corrected $=0.009 ; z=4.35$; Fig; 2B).

No significant metabolic change was found at 3 and 6 mo at this statistical level for the SPM analyses. No region of hypermetabolism was found at any time point.

\section{Progressive Atrophic Changes in Brain Structures on Serial MRI Scans After FPI}

Several structures showed volumetric changes after FPI: an increase in the ventricles $\left(F_{2,24}=4.99 ; P=0.03\right.$; Supplemental Fig. 3), a decrease in the ipsilateral cortex $\left(F_{2,24}=22.40, p<0.001\right.$, Fig. 3B $)$, and a decrease in the ipsilateral hippocampus $\left(F_{2,24}=13.16 ; P=0.001\right.$; Fig. $3 C)$. The effect of injury increased with time for the left cortex $\left(F_{2,24}=9.19 ; P<0.001\right)$ and the left hippocampus $\left(F_{2,24}=2.80 ; P=0.03\right)$.

No other significant changes were found in other ROIs. Whole-brain volumes were similar between FPI and sham rats $\left(F_{2,24}=0.001 ; P=0.97\right)$.

\section{Regional Changes in Hippocampal Shape After FPI}

HDM-LD demonstrated evolving changes in hippocampal shape, with the FPI group having greater regional hippocampal volume loss over time (Fig. 4; Supplemental Fig. 4). The maximum inward deformation distances increased progressively and bilaterally ( $1 \mathrm{wk}$ : left $=-0.18 \mathrm{~mm}$, right $=$ $-0.17 \mathrm{~mm} ; 1 \mathrm{mo}$ : left $=-0.17 \mathrm{~mm}$, right $=-0.21 \mathrm{~mm}$; $3 \mathrm{mo}$ : left $=-0.21 \mathrm{~mm}$, right $=-0.25 \mathrm{~mm}$; and $6 \mathrm{mo}$ : left $=-0.25 \mathrm{~mm}$, right $=-0.26 \mathrm{~mm}$ ). The pattern of regional hippocampal surface changes differed between the right and left hippocampi, and this difference also evolved over time after FPI.

\section{Assessment of Anxiety-Like Behavior and Learning}

Compared with sham rats, FPI rats were more anxious, with a lower percentage of time spent in the open arms of the EPM $\left(F_{1,27}=8.06 ; P<0.05\right)$ and fewer open-arm entries $\left(F_{1,27}=5.30 ; P<0.05\right)$. There was no confounding effect of locomotor activity in the FPI group, because there was no significant difference in the distance traveled in the $\operatorname{EPM}\left(F_{1,27}=1.40 ; P=0.24\right)$. Similarly, FPI rats spent less time $\left(F_{1,27}=5.87 ; P<0.05\right)$ and made fewer entries into the inner area $\left(F_{1,27}=14.66 ; P<0.0005\right)$ of the open field, indicative of increased anxiety (Supplemental Figs. 5A5D). Again, locomotor activity was not different between FPI and sham rats in the open field $\left(F_{1,27}=0.09 ; P=\right.$ 


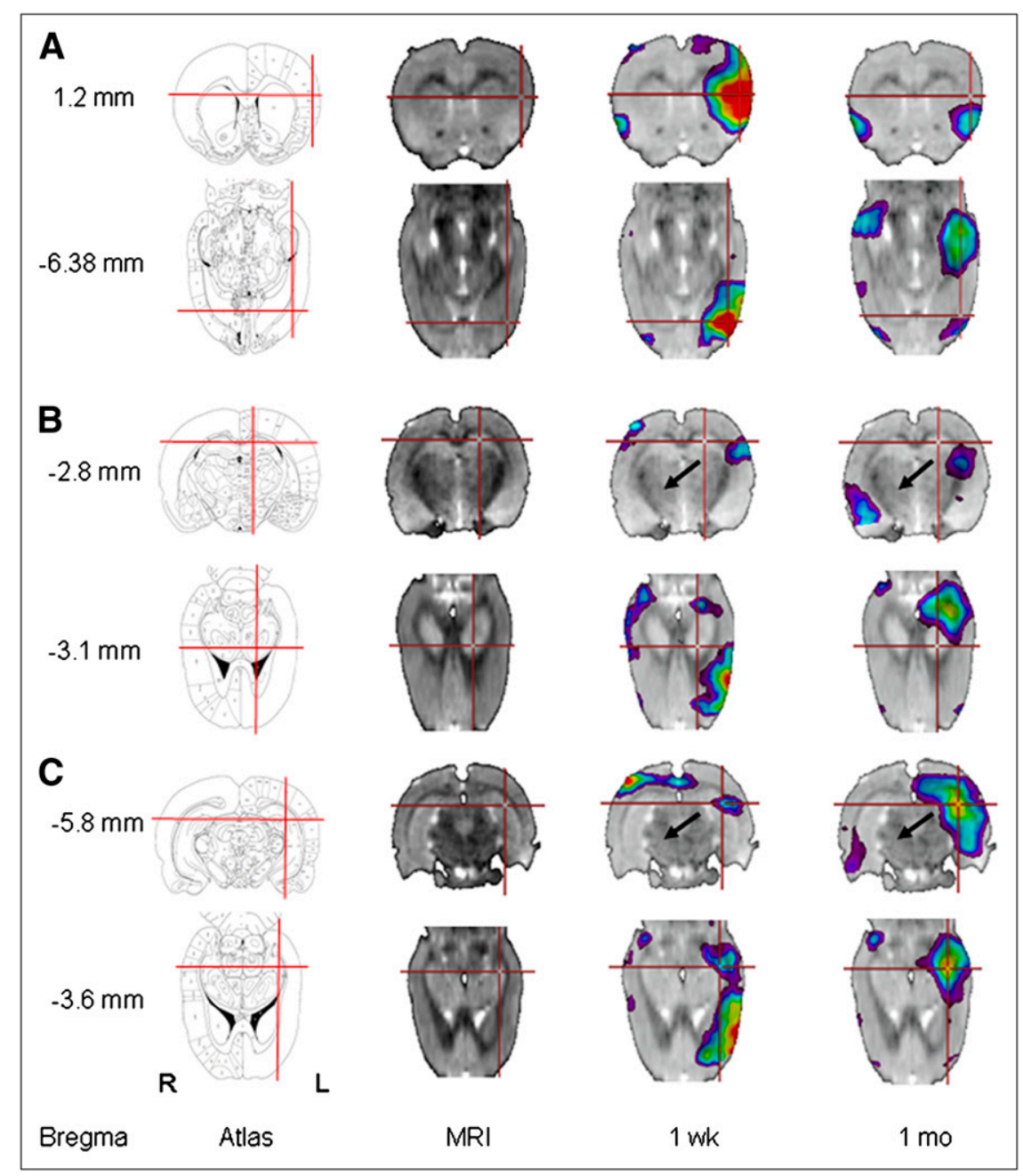

FIGURE 2. Statistical parametric maps of PET images demonstrating regions of hypometabolism at $1 \mathrm{wk}$ and $1 \mathrm{mo}$ after FPI superimposed on normalized baseline MR image in Anatomist (http://brainvisa.info). A-C correspond to different levels of brain on axial and corresponding horizontal sections, as depicted on atlas. Color scale ranges from height threshold ( $\mathrm{P}_{\mathrm{FDR}}$ corrected $<0.01$ ) to same level for all maps $(T=4.7)$. Arrows show site of trauma. No significant metabolic changes were found at 3 and 6 mo using ${ }^{18} \mathrm{~F}-\mathrm{FDG}$ PET at this statistical level. $R$ is right, $L$ is left.

0.77). No significant correlations were found between the magnitudes of the differences in the behavioral measures between the FPI and sham rats and the magnitudes of the differences in the imaging variables $(P>0.05$ in all cases).

No significant differences were found between FPI and sham rats for measures of learning and memory on the Morris water maze (data not shown).

\section{DISCUSSION}

This study investigated, in a comprehensive longitudinal paradigm, structural and functional changes that occur in the brain after closed-head TBI. We performed serial quantitative functional ${ }^{18} \mathrm{~F}-\mathrm{FDG}$ PET and morphometric MRI studies over 6 mo after lateral FPI in rats. The images were assessed quantitatively using a variety of analysis approaches: manual hypothesis-driven ROI analysis, automated deformation segmentations (HDM-LD), and wholebrain exploratory SPM. There were 4 major findings from this study. First, regions of hypometabolism in the brain were observed early after FPI, primarily affecting similar regions that showed subsequent progressive atrophic changes on MRI; these were maximal 1 mo after the injury but persisted for at least 6 mo in the ipsilateral amygdala. Second, there was discordance in the time course between the functional and structural changes-the former occurred earlier and decreased or resolved by 6 mo after FPI, and the latter were delayed in onset and continued to progress for at least 6 mo after FPI. Third, both hippocampi showed regional shape changes after FPI that evolved long after the trauma, with different patterns between the 2 sides. Fourth, the structural and functional changes are dynamic, appearing to consolidate between 3 and 6 mo after FPI, indicating the potential of an extended time window for application of neuroprotective strategies after FPI that may mitigate secondary neurodegenerative processes.

Serial ${ }^{18} \mathrm{~F}-\mathrm{FDG}$ PET images were analyzed using complementary approaches: ROI and SPM. ROI analysis has greater sensitivity in detecting changes but selectively samples regions on the basis of prior assumptions. In contrast, SPM samples the whole brain in an unbiased fashion but has lower sensitivity in detecting changes. Few previous 


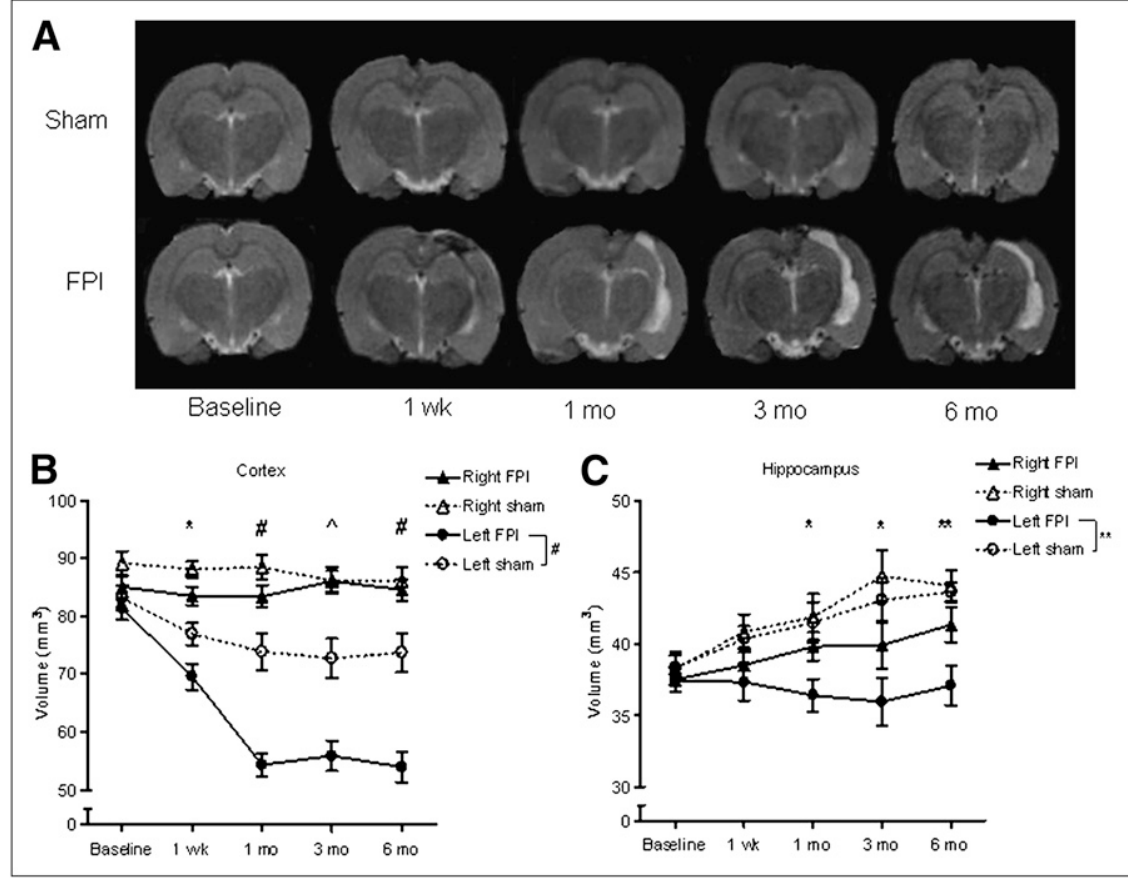

FIGURE 3. Progressive cerebral damage after FPI assessed using MRI. (A) Serial images from representative sham and FPI rats are shown at each time point. Time course of volume changes from baseline in cortex (B) and hippocampus (C) are shown. ${ }^{\star} P<0.05$. ${ }^{\star \star} P<0.005 . \wedge P<0.0005$. $\# P<$ 0.0001 . Mean \pm SEM. studies have applied SPM analysis to small-animal PET, and none involved models of TBI, despite the fact that the approach has been widely applied in human PET studies $(15,28,29)$. Many of the brain regions that showed early hypometabolism on ${ }^{18} \mathrm{~F}$-FDG PET images later showed progressive atrophy on serial MRI scans, particularly the cortex and hippocampi. This raises the possibility that the early hypometabolism may be mechanistically involved with induction of neurodegenerative processes after TBI. Compromised provision of energy substrates could result in oxidative stress that can induce cell death (30).

As shown in Figure 3A, areas of acute damage-induced injury are replaced by cerebrospinal fluid over time (31). Therefore, the possibility of partial-volume effects confounding the quantification of ${ }^{18} \mathrm{~F}-\mathrm{FDG}$ uptake on the PET scans in specific regions of the rat brain need to be considered. In particular, with the spatial normalization used for SPM, it is possible that the structural changes (atrophy) that occurred in the FPI group may have confounded the SPM analysis. However, the spatial and temporal patterns of the changes seen on the SPM analysis of the PET images argue strongly against this being a significant factor in the results. The regions of PET hypometabolism identified on the SPM analysis are prominent at the 1-wk and 1-mo scans after FPI, with this prominence tending to resolve over the subsequent scans. In contrast, the atrophy on the MR image is minimal at $1 \mathrm{wk}$ and continues to progress over the 6 mo after FPI. Also, relative hypometabolism was identified on the SPM analysis in regions for which there was no significant atrophy seen in the FPI rats, such as in the ventral and lateral cortex contralateral to the side of the injury. Further evidence of the validity of the SPM results is that they are consistent with the ROI analysis of the PET images, with the ROIs having been drawn on the co-

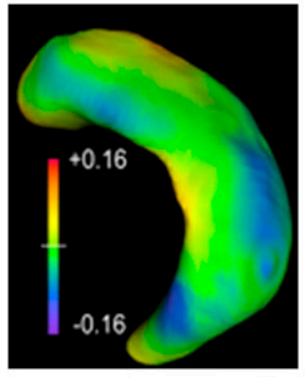

Baseline

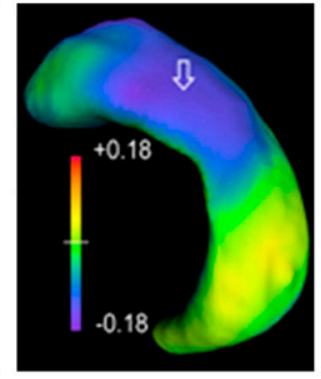

1 week

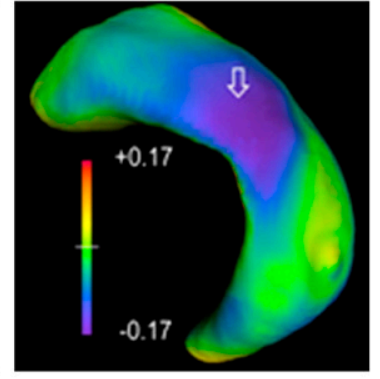

1 month

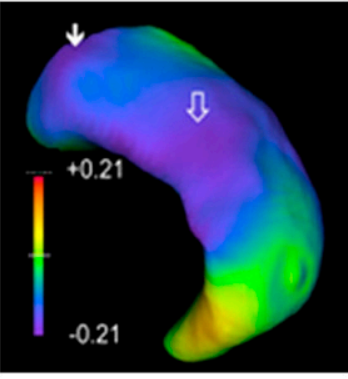

3 months

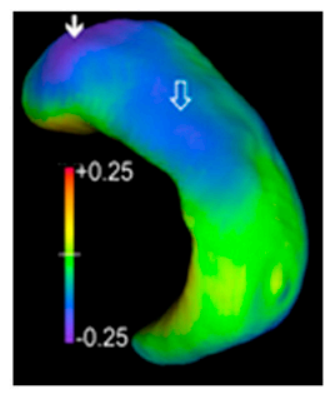

6 months

FIGURE 4. HDM-LD differences in left hippocampi between FPI and sham rats from serial MRI scans. Figure shows regional deformation distances for left hippocampi (ipsilateral to injury) in lateral and slightly caudal view. Open arrows in A show region of inward deformation primarily representing cornu ammonis 2, which becomes visible at $1 \mathrm{wk}$, progresses at 1 and 3 mo, and becomes less prominent at 6 mo. Closed arrow at 3 and 6 mo shows regions of accentuated volume loss, primarily representing cornu ammonis 1 (CA1). 
registered MRI scans and therefore accounting for any interindividual differences in the structure.

Another potential limitation of the PET analyses is the fact that baseline (preinjury) images were not acquired. However, unlike for the MR images, a relative, not absolute, quantification was performed on the PET images; therefore, the analysis examined differences between FPI and sham rats at each time point, not absolute changes over time in any of the groups. PET scans acquired from a rat before any procedures were performed would be highly unlikely to show any differences between those who were subsequently to receive FPI or sham injury (because littermates were randomly allocated to each of the intervention groups).

The SPM analysis demonstrated marked hypometabolism in the ipsilateral frontal and parietal cortices at $1 \mathrm{wk}$ after the trauma, extending subcortically to involve the striatum. These in vivo results are consistent with a previous report that used ex vivo 2-deoxyglucose autoradiography regional changes in brain metabolism in a cerebral contusion rat TBI model (32). In addition to the ipsilateral cortex changes, our study demonstrated a smaller region of change in the contralateral parietal cortex at $1 \mathrm{wk}$ after FPI (Fig. 2). Human studies have shown that a region, synaptically connected to a hypometabolic region and deep within the brain at the injury focus, also displays a decreased metabolic uptake (i.e., diaschisis) (33,34). Conversely, the absence of early metabolic change in the epicenter of the injury site in the cortex may be explained by early edema caused by the surgical procedure in both the sham and the FPI groups (7).

FPI rats display greater anxiety-like behavior than do sham rats, which persisted for at least 3 mo after FPI. The amygdala has been linked to anxiety disorders (35), but in this study no significant direct correlations were observed between the magnitude of anxiety-like behaviors and the structural or metabolic changes seen in the FPI rat brains.

ROI analysis and the automated HDM-LD method independently demonstrate a progressive decrease in hippocampal volume bilaterally after FPI, more marked on the side of the trauma. In addition, evolving surface changes in specific anatomic regions of the hippocampus in FPI rats were demonstrated. Different regions of the hippocampus along the septotemporal axis are composed of distinctly different subfields, which show changes according to the underlying pathophysiologic state. For example, the hippocampal surface anatomy shows different patterns in epilepsy, as compared with schizophrenia or depression (23). Our current findings show dynamic changes in hippocampal surface structure, likely reflecting the complex underlying evolution of multifactorial pathophysiologic changes over time after TBI.

Although multiple regions of the hippocampal surface were affected, the surface changes found after FPI using HDM-LD mapping were most prominent over the CA1 subfield of the hippocampus, particularly at the later time points, supporting previous clinical studies that have suggested a particular sensitivity of CA1 to TBI (36). CA1 and all the anterior hippocampus comprise greater excitatory cell density and lower inhibitory cell density (37), making the hippocampal head more susceptible to the excitotoxic effects of TBI. Supporting this fact, the nonhuman primate frontobasal cortex receives around $70 \%$ of the projections from the hippocampal head (38). Therefore, the cortical damage demonstrated in this study may result in hippocampal head deeferentation and consequently in hippocampal cell death.

\section{CONCLUSION}

This study used serial multimodality in vivo imaging and a variety of complementary image analytic techniques to characterize the temporal development and regional distribution of the long-term structural and functional changes that occur in the brain after the FPI model of closed-head TBI in rats. The results demonstrated widespread hypometabolic changes affecting key brain structures, particularly the hippocampus and cortex, that are present as early as $1 \mathrm{wk}$ after FPI. Progressive atrophic structural changes then occured which evolved over the next 3-6 mo. The finding that the cerebral hypometabolism predates the progressive cerebral atrophy suggests that it may play a mechanistic role in the subsequent neurodegeneration in affecting these brain regions. The temporal evolution of structural and functional brain changes demonstrated in this study may have implications for the pathophysiology of adverse long-term neurologic and neuropsychiatric consequences commonly seen in animals and humans after TBI, and suggest a potential window for the application of therapeutic interventions.

\section{ACKNOWLEDGMENT}

We thank Prof. Gary Egan and the staff of the Small Animal MRI Facility, Howard Florey Institute, for assistance with MRI scanning. We also thank the radiochemistry staff of the Peter MacCallum Cancer Centre for assistance with ${ }^{18} \mathrm{~F}$ FDG PET. This work was supported by the Victorian Government Transport Accident Commission (TAC) in the form of a Victorian Neurotrauma Initiative Project grant (DP0023) and a project grant from the Australian NHMRC (400088).

\section{REFERENCES}

1. Bergsneider M, Hovda DA, Lee SM, et al. Dissociation of cerebral glucose metabolism and level of consciousness during the period of metabolic depression following human traumatic brain injury. J Neurotrauma. 2000;17:389-401.

2. Bergsneider M, Hovda DA, Shalmon E, et al. Cerebral hyperglycolysis following severe traumatic brain injury in humans: a positron emission tomography study. J Neurosurg. 1997;86:241-251.

3. Yamaki T, Yoshino E, Fujimoto M, Ohmori Y, Imahori Y, Ueda S. Chronological positron emission tomographic study of severe diffuse brain injury in the chronic stage. J Trauma. 1996;40:50-56.

4. Bergsneider M, Hovda DA, McArthur DL, et al. Metabolic recovery following human traumatic brain injury based on FDG-PET: time course and relationship to neurological disability. J Head Trauma Rehabil. 2001;16:135-148.

5. Belanger HG, Vanderploeg RD, Curtiss G, Warden DL. Recent neuroimaging techniques in mild traumatic brain injury. J Neuropsychiatry Clin Neurosci. 2007; 19:5-20 
6. Moore AH, Osteen CL, Chatziioannou AF, Hovda DA, Cherry SR. Quantitative assessment of longitudinal metabolic changes in vivo after traumatic brain injury in the adult rat using FDG-microPET. J Cereb Blood Flow Metab. 2000;20:1492-1501.

7. Bouilleret V, Cardamone L, Liu YR, Fang K, Myers DE, O'Brien TJ. Progressive brain changes on serial manganese-enhanced MRI following traumatic brain injury in the rat. $J$ Neurotrauma. 2009;26:1999-2013.

8. Immonen RJ, Kharatishvili I, Niskanen JP, Grohn H, Pitkanen A, Grohn OH. Distinct MRI pattern in lesional and perilesional area after traumatic brain injury in rat: 11 months follow-up. Exp Neurol. 2009;215:29-40.

9. Kharatishvili I, Immonen R, Grohn O, Pitkanen A. Quantitative diffusion MRI of hippocampus as a surrogate marker for post-traumatic epileptogenesis. Brain. 2007; $130: 3155-3168$.

10. Thompson HJ, Lifshitz J, Marklund N, et al. Lateral fluid percussion brain injury: a 15-year review and evaluation. J Neurotrauma. 2005;22:42-75.

11. Pitkanen A, McIntosh TK. Animal models of post-traumatic epilepsy. J Neurotrauma. 2006;23:241-261.

12. Jones NC, Cardamone L, Williams JP, Salzberg MR, Myers D, O'Brien TJ. Experimental traumatic brain injury induces a pervasive hyperanxious phenotype in rats. J Neurotrauma. 2008;25:1367-1374.

13. McIntosh TK, Vink R, Noble L, et al. Traumatic brain injury in the rat: characterization of a lateral fluid-percussion model. Neuroscience. 1989;28:233-244.

14. Jupp B, O'Brien TJ. Application of coregistration for imaging of animal models of epilepsy. Epilepsia. 2007;48(suppl 4):82-89.

15. Casteels C, Vermaelen P, Nuyts J, et al. Construction and evaluation of multitracer small-animal PET probabilistic atlases for voxel-based functional mapping of the rat brain. J Nucl Med. 2006;47:1858-1866.

16. Bouilleret V, Hogan RE, Velakoulis D, et al. Morphometric abnormalities and hyperanxiety in genetically epileptic rats: a model of psychiatric comorbidity? Neuroimage. 2009;45:267-274.

17. Hogan RE, Bouilleret V, Liu YR, et al. MRI-based large deformation high dimensional mapping of the hippocampus in rats: development and validation of the technique. J Magn Reson Imaging. 2009;29:1027-1034.

18. Gardner R, Hogan RE. Three-dimensional deformation-based hippocampal surface anatomy, projected on MRI images. Clin Anat. 2005;18:481-487.

19. Csernansky JG, Joshi S, Wang L, et al. Hippocampal morphometry in schizophrenia by high dimensional brain mapping. Proc Natl Acad Sci USA. 1998;95:11406-11411.

20. Hogan RE, Carne RP, Kilpatrick CJ, et al. Hippocampal deformation mapping in MRI negative PET positive temporal lobe epilepsy. J Neurol Neurosurg Psychiatry. 2008;79:636-640.

21. Hogan RE, Wang L, Bertrand ME, et al. MRI-based high-dimensional hippocampal mapping in mesial temporal lobe epilepsy. Brain. 2004;127:1731-1740.

22. Posener JA, Wang L, Price JL, et al. High-dimensional mapping of the hippocampus in depression. Am J Psychiatry. 2003;160:83-89.

23. Hogan RE, Wang L, Bertrand ME, et al. Predictive value of hippocampal MR imaging-based high-dimensional mapping in mesial temporal epilepsy: preliminary findings. AJNR. 2006;27:2149-2154.
24. Paxinos G, Watson C. The Rat Brain in Stereotaxic Coordinates. 4th ed. San Diego, CA: Academic Press; 1998.

25. Carobrez AP, Bertoglio LJ. Ethological and temporal analyses of anxiety-like behavior: the elevated plus-maze model 20 years on. Neurosci Biobehav Rev. 2005; 29:1193-1205

26. Prut $\mathrm{L}$, Belzung $\mathrm{C}$. The open field as a paradigm to measure the effects of drugs on anxiety-like behaviors: a review. Eur J Pharmacol. 2003;463:3-33.

27. Jones NC, Kumar G, O’Brien TJ, Morris MJ, Rees SM, Salzberg MR. Anxiolytic effects of rapid amygdala kindling, and the influence of early life experience in rats. Behav Brain Res. 2009;203:81-87.

28. Soto-Montenegro ML, Vaquero JJ, Pascau J, Gispert JD, Garcia-Barreno P, Desco M. Detection of visual activation in the rat brain using 2-deoxy-2- $\left[{ }^{18} \mathrm{~F}\right]$ fluoro-D-glucose and Statistical Parametric Mapping (SPM). Mol Imaging Biol. 2009;11:94-99.

29. van Kuyck K, Casteels C, Vermaelen P, Bormans G, Nuttin B, Van Laere K. Motor- and food-related metabolic cerebral changes in the activity-based rat model for anorexia nervosa: a voxel-based microPET study. Neuroimage. 2007; 35:214-221.

30. Loh KP, Huang SH, De Silva R, Tan BK, Zhu YZ. Oxidative stress: apoptosis in neuronal injury. Curr Alzheimer Res. 2006;3:327-337.

31. Albensi BC, Knoblach SM, Chew BG, O'Reilly MP, Faden AI, Pekar JJ. Diffusion and high resolution MRI of traumatic brain injury in rats: time course and correlation with histology. Exp Neurol. 2000;162:61-72.

32. Yoshino A, Hovda DA, Kawamata T, Katayama Y, Becker DP. Dynamic changes in local cerebral glucose utilization following cerebral conclusion in rats: evidence of a hyper- and subsequent hypometabolic state. Brain Res. 1991;561: $106-119$.

33. Chassoux F, Semah F, Bouilleret V, et al. Metabolic changes and electro-clinical patterns in mesio-temporal lobe epilepsy: a correlative study. Brain. 2004;127: 164-174.

34. Ryvlin P, Bouvard S, Le Bars D, et al. Clinical utility of flumazenil-PET versus $\left[{ }^{18} \mathrm{~F}\right]$ fluorodeoxyglucose-PET and MRI in refractory partial epilepsy: a prospective study in 100 patients. Brain. 1998;121:2067-2081.

35. Etkin A, Prater KE, Schatzberg AF, Menon V, Greicius MD. Disrupted amygdalar subregion functional connectivity and evidence of a compensatory network in generalized anxiety disorder. Arch Gen Psychiatry. 2009;66:1361-1372.

36. Kotapka MJ, Gennarelli TA, Graham DI, et al. Selective vulnerability of hippocampal neurons in acceleration-induced experimental head injury. $J$ Neurotrauma. 1991;8:247-258.

37. Bernasconi N, Bernasconi A, Caramanos Z, Antel SB, Andermann F, Arnold DL. Mesial temporal damage in temporal lobe epilepsy: a volumetric MRI study of the hippocampus, amygdala and parahippocampal region. Brain. 2003;126: 462-469.

38. Cavada C, Company T, Tejedor J, Cruz-Rizzolo RJ, Reinoso-Suarez F. The anatomical connections of the macaque monkey orbitofrontal cortex: a review. Cereb Cortex. 2000;10:220-242. 